\title{
Topical Celecoxib
}

National Cancer Institute

\section{Source}

National Cancer Institute. Topical Celecoxib. NCI Thesaurus. Code C148519.

A topical cream formulation containing celecoxib, a nonsteroidal anti-inflammatory drug (NSAID), with anti-inflammatory and potential keratolytic, chemopreventive and antineoplastic activities. Upon topical application to the affected area, celecoxib selectively binds to and inhibits cyclooxygenase-2 activity (COX-2), which may result in localized keratinocyte apoptosis. The breakdown of keratinocytes prevents their proliferation locally and may reduce tumor cell proliferation. 